SCIENTIFIC CORRESPONDENCE

\title{
Prediction of visual outcome after retinal detachment surgery using the Lotmar visometer
}

\author{
B Yazıcı, Ö Gelişken, R Avcı, A Yücel
}

Br J Ophthalmol 2002;86:278-281

Aim: To evaluate whether an achromatic interferometer, the Lotmar visometer, is useful in predicting postoperative visual outcome in patients with primary rhegmatogenous retinal detachment (RD) involving the macula.

Methods: This prospective study included 40 eyes of 40 non-consecutive patients with macula-off RD. The eyes were phakic or pseudophakic, had a clear optical media, and had a measurable potential vision on preoperative visometric examination. Preoperative variables included Snellen visual acuity, duration of macular detachment, extent of RD, and visometric potential acuity. Reattachment surgery consisted of radial scleral buckling in 33 patients, circumferential scleral buckling and encircling in seven patients, and subretinal fluid drainage in 10 patients. Retinal breaks were treated with cryotherapy or laser photocoagulation. Patients were followed up for at least 6 months after uncomplicated surgery. Best corrected visual acuity measured at any time during follow up was correlated with the preoperative variables.

Results: Preoperative visual acuity was less than 20/200 in $37(93 \%)$ of 40 patients. Potential visual acuity of $20 / 200$ or better was measured using the Lotmar visometer in 37 patients (93\%). Postoperative visual acuity was correlated significantly with duration of macular detachment $(r=0.55 ; p<0.001)$, and extent of RD approached statistical significance $(r=0.31 ; p=0.05)$. There was a higher correlation between postoperative visual acuity and the visometric measurements $(r=0.61 ; p<0.001)$.

Conclusions: The Lotmar visometer may be a valuable method to estimate visual outcome after uncomplicated scleral buckling surgery in patients with RD involving the macula

A lthough scleral buckling surgery achieves a high anatomical success rate in patients with rhegmatogenous etinal detachment (RD), the visual recovery remains less satisfactory, particularly when the macula is involved. Several studies ${ }^{1-5}$ have reported that preoperative visual acuity, duration of macular detachment, and extent of RD are the major factors related to postoperative visual outcome. However, it is generally difficult to estimate visual prognosis using these variables in clinical practice. Friberg and Eller $^{6}$ used the potential acuity meter (PAM) to predict postoperative visual improvement in patients with $\mathrm{RD}$ and found a high correlation between preoperative PAM measurements and postoperative visual outcome. We used the Lotmar visometer in a series of patients with macula-off RD to determine if this method is useful in predicting postoperative visual acuity.

The Lotmar visometer, which was developed by Lotmar ${ }^{7}$ in 1980 for quantitative assessment of visual capacity independent of ocular media opacities, is an interferometric method using a white light source. Two separate coherent light beams directed through the pupil overlap inside the eye and produce interference fringes seen as light and dark stripes. Visual acuity is measured by varying the width of the stripe patterns corresponding to Snellen equivalents.

\section{MATERIALS AND METHODS}

Between 1 June 1995 and 31 July 1997, 63 patients with primary rhegmatogenous RD involving the macula who underwent scleral buckling surgery were enrolled in this study. Phakic or pseudophakic eyes with posterior chamber intraocular lens implants, in which the optical media is clear, were included. Patients with giant retinal tears, coexisting ocular disease, or a history of previous retinal surgery were excluded.

We eliminated 13 patients who could not see any interferometric stripes on the visometric examination. An additional 10 patients were excluded after surgery because of surgical failure (five patients), postoperative complications that could adversely affect visual prognosis (preretinal fibrosis in two patients and nuclear cataract in one patient), and having less than 6 months of follow up (three patients). Therefore, the study included 40 eyes of 40 patients who were followed up for at least 6 months after a successful scleral buckling surgery. Patient ages ranged from 21 to 73 years (mean 52.7 years). Retinal detachments were associated with pseudophakia in five eyes and with high myopia in five eyes. This represents a non-consecutive series of patients; however, no other inclusion or exclusion criteria were applied.

Duration of macular detachment was determined by the patient's recall of the duration of central blurring or vision loss and was recorded in weeks. Preoperative visual acuity was measured using a Snellen chart under standardised conditions. The fundus was examined with a three mirror Goldmann lens, and a detachment chart was drawn.

Potential visual acuity was measured by the same physician (BY) using the Lotmar visometer (Haag-Streit, Bern, Switzerland) the day before the operation. The measurements were done in a dark room after full dilatation of the pupil. The visometer was mounted on a slit lamp. Light aperture of 0.5 $\mathrm{mm}$, visual field of $3.5^{\circ}$, and voltage of $6.0 \mathrm{~V}$ were set on the device. Before the measurement, patients were instructed about the method. Their normal eyes were tested first to familiarise them with the interference stripes. The light beam was then directed onto the pupil in the eye with RD. The stripes corresponding to visual acuity of 20/800 were presented and the direction of the stripes was asked of the patient. When the patient reported seeing these stripes and indicated their directions properly, the stripes were gradually narrowed. The narrowest set of stripes that the patient correctly saw in different directions was recorded as potential visual acuity.

Reattachment surgery consisted of scleral buckling using radial placement of a silicone sponge in 33 patients and

Abbreviations: PAM, potential acuity meter; RD, retinal detachment 
Table 1 Data related to preoperative variables and postoperative visual acuity $(n=40)$ *

\begin{tabular}{lll}
\hline Variable & Mean & Range \\
\hline Duration of macular detachment (weeks) & $5.00(7.4)$ & $1.0-36.0$ \\
Extent of RD (hours) & $7.30(2.4)$ & $4.0-12.0$ \\
Preoperative VA & $2.03(0.7)$ & $3.0-0.1$ \\
Visometric VA & $0.66(0.3)$ & $1.3-0.1$ \\
Postoperative VA & $0.46(0.3)$ & $1.3-0.1$
\end{tabular}

*RD = retinal detachment; $V A=$ visual acuity (logMAR unit)

Numbers in parentheses are standard deviations.

Table 2 Preoperative visometric potential acuities versus postoperative visual acuities $(n=40)$

\begin{tabular}{llll}
\hline \multirow{2}{*}{$\begin{array}{l}\text { Visometric } \\
\text { potential acuity }\end{array}$} & \multicolumn{2}{l}{ Postoperative visual acuity } \\
\cline { 2 - 4 } & $20 / 400-20 / 100$ & 20/80-20/65 & 20/50 or higher \\
\hline $20 / 400-20 /$ & 8 & 5 & 6 \\
100 & 3 & 10 \\
$20 / 80-20 / 65$ & 1 & - & 7 \\
$20 / 50$ or higher & - & &
\end{tabular}

circumferential placement of a solid silicone combined with an encircling band in seven patients. Subretinal fluid was drained in 10 patients. Retinopexy was performed by either transscleral cryotherapy during surgery or laser photocoagulation after surgery in all patients.

After surgery, different ophthalmologists without knowledge of the visometric predictions measured visual acuity after 1 week, after 1 month, and then every 3 months for 1 year. Patients were then examined every 6 months. The best visual acuity with refractive correction obtained at any time during follow up was used for statistical analyses. Mean follow up was 18.9 months (range 6-46 months).

We transformed all Snellen and visometric values into their logarithm of the minimum angle of resolution (logMAR) equivalents for statistical purposes. Visual acuity at the level of counting fingers was converted to a Snellen equivalent by writing the counting fingers distance as the numerator and 200 as the denominator. A logMAR value of 3.0 was assigned for visual acuity on the level of hand movements. ${ }^{6}$

Linear and multiple regression analyses were performed to determine the correlation of the visometric measurement and the other three preoperative variables (preoperative visual acuity, duration of macular detachment, and extent of RD) with best corrected postoperative visual acuity.

\section{RESULTS}

Preoperative Snellen visual acuity was less than 20/200 in 37 (93\%) of 40 patients and less than or equal to counting fingers from 1 metre in 28 patients (70\%) (Table 1). Visometric examination revealed a potential acuity equal to $20 / 200$ or better in 37 patients $(93 \%)$.

After surgery, best corrected visual acuity was 20/50 or better in 23 patients (58\%). The mean time for measuring the best corrected visual acuity was 9.9 months (range 1-35 months) after surgery. Postoperative visual acuity was better than visometric potential acuity in 32 patients $(80 \%)$. Of 19 patients who had a potential acuity of $20 / 100$ or less, six $(32 \%)$ obtained a postoperative visual acuity of $20 / 50$ or better. However, postoperative visual acuity was better than 20/50 in 17 $(81 \%)$ of 21 patients who had a potential acuity of $20 / 80$ or greater (Table 2 ).

In linear analysis, postoperative visual acuity was correlated significantly with duration of macular detachment and extent

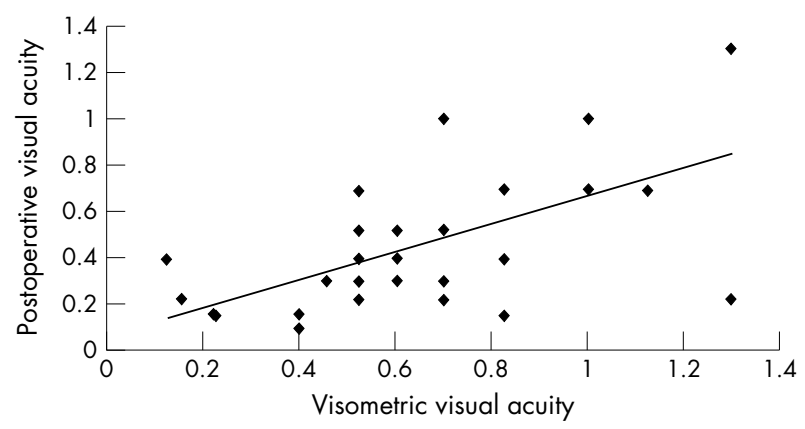

Figure 1 Scattergram showing the correlation between preoperative visometric acuity and postoperative visual acuity in $\mathbf{4 0}$ eyes undergoing successful retinal detachment surgery (both in $\log M A R$ units) $(r=0.61, p<0.001)$.

of $\mathrm{RD}(r=0.55 ; \mathrm{p}<0.001$ and $r=0.31 ; \mathrm{p}=0.05$, respectively $)$. We found a higher correlation between the visometric potential acuity and the postoperative visual acuity $(r=0.61 ; \mathrm{p}<0.001)$ (Fig 1). There was no correlation between preoperative and postoperative visual acuities $(r=0.05 ; \mathrm{p}>0.05)$. Multiple regression analysis, using the three preoperative clinical variables to predict the postoperative visual result, yielded a correlation coefficient of $0.61 \quad(p<0.001)$, which is equal to the value obtained using only the visometric prediction.

\section{DISCUSSION}

The critical factor determining visual prognosis in patients with RD is whether the macula is detached before the operation. Most patients present after macular detachment has caused central visual loss, and $37 \%$ to $59 \%$ of these can regain $20 / 50$ or better visual acuity after successful treatment. ${ }^{3589}$ Multiple factors related to preoperative and postoperative observations and surgical methods are correlated significantly with visual outcome-for example, preoperative visual acuity, duration of macular detachment, extent of RD, patient age, status of crystalline lens, height of macular detachment, location and size of retinal break, proliferative vitreoretinopathy, preoperative ocular hypotony or hypertension, drainage or non-drainage of subretinal fluid, number of cryo applications, extent of indentation, encircling or not circling, and the length of follow up..$^{2-5} 9$ A regression formula that can deal with these variables simultaneously might be helpful in predicting the postoperative vision. Burton and Lambert ${ }^{11}$ identified 26 of 200 observations that significantly affect visual prognosis and developed a mathematical model that predicts in a broad range of visual acuity to approximately $67 \%$ of accuracy. Although it is possible to predict visual capacity using this method, it might not be practical for widespread clinical use.

Preoperative visual acuity, duration of macular detachment, and extent of $\mathrm{RD}$ are the main variables correlating significantly with postoperative visual result and are evaluated routinely on preoperative examination. We compared the predictive value of these clinical variables with that of visometric potential acuity. Increased duration of macular detachment and extent of RD were significantly associated with less postoperative visual acuity ( $r=0.55$ and $r=0.31$, respectively). We found a higher correlation between visometric potential acuity and postoperative visual acuity than the other variables $(r=0.61)$. According to our findings, a patient with potential acuity of 20/80 or better might reach a postoperative acuity of $20 / 50$ or better, with a possibility of $81 \%$. When potential acuity is $20 / 100$ or less, the possibility reduces to $32 \%$ (Table 2 ).

Friberg and Eller, ${ }^{6}$ who used the PAM in 50 patients, found that the preoperative PAM measurement was correlated better with final visual acuity than with any other variable $(r=0.72)$. They found the highest correlation between potential acuity and visual improvement $(r=0.92)$. According to their findings, 
a patient with poor preoperative Snellen acuity who has a good PAM result is likely to obtain a large visual improvement after surgery.

Although our study was designed similar to that of Friberg and Eller, ${ }^{6}$ there are some differences between the two studies. They included 17 patients who were unable to read any letter on the PAM and presumed that these patients had a potential acuity of 20/800. We excluded patients who could not see the interference lines on visometric examination. They considered the final visual acuity to be that measured 6 months after surgery. We defined this variable as the highest visual acuity measured at any time during follow up. Visual acuity may continue to improve up to 2 years after surgery, particularly in young and myopic patients. ${ }^{13}{ }^{14}$ The Lotmar visometer usually underestimates potential acuity and might show better agreement with visual acuities measured in the postoperative 6 months.

In another study, Yasukawa et $a l^{15}$ used the PAM and laser interferometer for predicting the visual outcome in 31 patients with macular involvement. They found that postoperative visual acuity correlated better with the laser interferometry and PAM results ( $r=0.73$ and $r=0.71$, respectively) than with preoperative visual acuity.

Of the several factors, preoperative visual acuity is the most important variable related to the final visual result after anatomically successful reattachment surgery. ${ }^{10}$ In a series of patients with macular involvement and preoperative visual acuity of $20 / 200$ or better, postoperative acuity of 20/50 or better was obtained in $65 \%$ of eyes. ${ }^{5}$ However, the chance of regaining postoperative acuity of $20 / 50$ or better diminishes to $31 \%$ when preoperative acuity is less than 20/200. Most large series of $\mathrm{RD}$ involving the macula report that preoperative acuity is less than $20 / 200$ in about $80 \%$ of cases. ${ }^{13-5}$ In our series, preoperative visual acuity was worse than 20/200 in 37 patients (92\%). The value of preoperative vision in predicting visual capacity might be limited in this subgroup of patients.

With regard to duration of macular detachment, it is well known that longer duration of detachment is associated with less visual recovery. However, the data about duration of macular detachment significantly affecting postoperative vision are conflicting. The critical duration has been reported as 1 week, 1 month, and 2 months. ${ }^{25}{ }^{16}$ Furthermore, duration of macular detachment is determined subjectively, according to the patient's recall, and might not be reliable, particularly in patients with longstanding RD.

As with the PAM and laser interferometer, the Lotmar visometer has been used to measure the visual capacity in eyes with opacity of ocular media, particularly in cataracts. ${ }^{17}{ }^{18} \mathrm{~A}$ Snellen chart is projected into the eye via a narrow beam of light in the PAM, a series of light and dark stripes formed by interference of two light beams in the visometer. The visometer is as accurate as the PAM in determining visual capacity in patients with cataracts. ${ }^{18}$ In earlier studies, these methods were considered to be unreliable in measuring visual capacity in eyes with media opacities and macular pathological features. Predicted value was better than the attained postoperative visual acuity when there was cystic oedema, serous detachment, or hole or age related degeneration of the macula. ${ }^{17}{ }^{19} 20$ In these conditions, the disparity between these two measurements probably represents a measure of the potential vision if the underlying macular disease can be treated.$^{21}$ The visual improvement obtained after treatment of cystic macular oedema secondary to posterior uveitis or cataract surgery and after the surgery of macular hole correlated well with pretreatment potential acuity measurements. ${ }^{21-23}$

In our study, 37 patients who could not read any letter on the Snellen chart had a potential visual acuity of 20/400 or better on visometric examination. This discrepancy deserves an explanation. The beam light of potential acuity meters illuminates a narrower area on the macula than the light reflected off a Snellen chart, and, therefore, the light scatter caused by intraretinal microcystic oedema may be less. ${ }^{64}$ In addition to histopathological changes, refractive changes associated with topographical changes in the detached macula also decrease visual acuity significantly. Interferometers or PAMs can measure potential acuity because they create images on the retina without the influence of the refraction within certain limits. ${ }^{7} 1825$

Some patients could not find the light at all on the visometer, although they were able to see the light of the examination room and more. We noticed that the macular detachment was higher and the fovea was considerably away from the optical axis in these patients. The increased macular elevation may be associated with a large refractive error and less visual capacity ${ }^{10}{ }^{16}$ Although some patients stated that the stripes are not straight and regular on visometric examination, this did not prevent them seeing the stripes and describing their directions. Such an optical distortion might affect the predictions negatively in the devices using letter charts. A study found that compared with other type of potential acuity devices, interference fringe-type instruments tended to give high values of prediction in patients with macular disease. ${ }^{26}$

Visometric potential acuity was higher than postoperative acuity in four patients in our study (one line on the Snellen chart in three patients and three lines in the other patient). Retinal reattachment was obtained by local scleral indentation in these patients, and the macula appeared normal after surgery. We do not know why visual improvement was less than predicted in these patients.

In conclusion, although having some limitations, potential acuity measurement using the Lotmar visometer may be a useful method in predicting visual outcome in RD with macular involvement. This method may be used to inform patients of their visual capacity. Because of the multitude of variables associated with postoperative visual acuity, it has been difficult to compare visual results obtained by several RD surgery techniques. ${ }^{27}$ Comparisons based on potential acuity measurements might help overcome this difficulty.

\section{ACKNOWLEDGEMENTS}

The authors have no commercial or proprietary interest in any of the equipment mentioned in this study.

\section{Authors' affiliations}

B Yazıcı, Ö Gelişken, R Avcı, A Yücel, Department of Ophthalmology, Uludag University School of Medicine, Bursa, Turkey

Correspondence to: Bülent Yazıcı, MD, Department of Ophthalmology, Uludag University School of Medicine, Gorukle Bursa 16059, Turkey; byazici@uludag.edu.tr

Accepted for publication 25 September 2001

\section{REFERENCES}

1 Wilkinson CP. Pseudophakic retinal detachments. Retina 1985;5:1-4.

2 Grupposo SS. Visual acuity following surgery for retinal detachment. Arch Ophthalmol 1975;93:327-30.

3 Burton TC. Recovery of visual acuity after retinal detachment involving the macula. Trans Am Ophthalmol Soc 1982;80:475-97.

4 McPherson AR, O'Malley RE, Butner RW, et al. Visual acuity after surgery for retinal detachment with macular involvement. Ann Ophthalmol 1982;14:639-45.

5 Tani P, Robertson DM, Langworthy A. Prognosis for central vision with anatomic reattachment in rhegmatogenous retinal detachment with macula detached. Am J Ophthalmol 1981;92:611-20.

6 Friberg TR, Eller AW. Prediction of visual recovery after scleral buckling of macula-off retinal detachments. Am J Ophthalmol 1992;1 14:715-22.

7 Lotmar V. Apparatus for the measurement of retinal visual acuity by moire fringes. Invest Ophthalmol Vis Sci 1980;1 9:393-400.

8 Wilkinson CP, Bradford RH. Complications of draining subretinal fluid. Retina 1984;4:1-4

9 Ross WH, Kozy DW. Visual recovery in macula-off rhegmatogenous retinal detachments. Ophthalmology 1998;105:2149-53.

10 Michels $\mathbf{R}$, Wilkinson CP, Rice TA. Results of retinal reattachment surgery. In: Michels R, Wilkinson CP, Rice TA, eds. Retinal detachment. St Louis: CV Mosby, 1990:917-58. 
11 Burton TC, Lambert RW. A predictive model for visual recovery following retinal detachment surgery. Ophthalmology 1978;85:619-25

12 Kaufmann PL. Prognosis of primary rhegmatogenous retinal detachments, 2: accounting for and predicting final visual acuity in surgically reattached cases. Acta Ophthalmol 1976:54:61-74.

13 Markham RH, Chignell AH. Visual acuity after retinal detachment operations. Trans Ophthalmol Soc U K 1979;99:197-200.

14 Kreissig I, Simader E, Fahle M, et al. Visual acuity after segmental buckling and non-drainage: a 15-year follow-up. Eur J Ophthalmol 1995:5:240-6.

15 Yasukawa T, Fukuda T, Kishimoto $M$, et al. Prediction of postoperative visual acuity in retinal detachment with macular involvement. Nippon Ganka Gakkai Zasshi 1995;99:318-22.

16 Kreissig I. Prognosis of return of macular function after retinal reattachment. Mod Probl Ophthalmol 1977; 18:415-29.

17 Bernth-Petersen P, Naeser K. Clinical evaluation of the Lotmar Visometer for macula testing in cataract patients. Acta Ophthalmol 1982;60:525-32.

18 Spurny RC, Zaldivar R, Belcher CD, et al. Instruments for predicting visual acuity: a clinical comparison. Arch Ophthalmol 1986; 104: 196-200.
19 Faulkner W. Laser interferometric prediction of postoperative visual acuity in patients with cataracts. Am J Ophthalmol 1983;95:626-36. 20 Bloom TD, Fishmann GA. Laser interferometric visual acuity in senile macular degeneration. Arch Ophthalmol 1983;101:925-6.

21 Palestine AG, Alter GJ, Chan CC, et al. Laser interferometry and visual prognosis in uveitis. Ophthalmology 1985;92:1567-9.

22 Sinskey RM, Stoppel JO. Potential acuity meter and visual outcome in pseudophakic eyes with clinical cystoid macular edema. Eur J Implant Refract Surg 1994;6:6-9.

23 Smiddy WE, Thomley ML, Knighton RW, et al. Use of potential acuity meter and laser interferometer to predict visual acuity after macular hole meter and laser interferometer to
surgery. Retina 1994;14:305-9.

24 Guyton DL. Misleading predictions of postoperative visual acuity [editorial]. Arch Ophthalmol 1986;104:189.

25 Enoch JM, Bedell HE, Kaufmann HE. Interferometric visual acuity testing in anterior segment disease. Arch Ophthalmol 1979;97:1916-9.

26 Fish GE, Birch DG, Fuller DG, et al. A comparison of visual function tests in eyes with maculopathy. Ophthalmology 1986;93:1 177-82.

27 Wilkinson CP. What is the "best" way to fix a routine retinal detachment? In: Lewis H, Ryan SJ, eds. Medical and surgical retina. St Louis: Mosby, 1994:85-102.

\section{Rapid responses}

Letters on the following British Journal of Ophthalmology papers have been published recently as rapid responses on the $B J O$ website. To read these letters visit www.bjophthalmol.com and click on "Read eletters"

A Joseph, H S Dua, A J King. Failure of amniotic membrane transplantation in the treatment of acute ocular burns. Br J Ophthalmol 2001;85:1065-9.

D Paridaensa, H Beekhuisb, W van den Boscha, et al. Amniotic membrane transplantation in the management of conjunctival malignant melanoma and primary acquired melanosis with atypia. Br J Ophthalmol 2001;85:658-61.

$M$ Cahill, $\mathrm{P}$ Eustace, $\mathrm{V}$ de Jesus. Pupillary autonomic denervation with increasing duration of diabetes mellitus. Br J Ophthalmol 2001;85:1225-30.

K Miyata, T Takahashi, A Tomidokoro, et al. Iatrogenic keratectasia after phototherapeutic keratectomy. Br J Ophthalmol 2001;85:238.

$\mathrm{H}$ Ahmed, R Y Hassan, U H Pindiga. Xeroderma pigmentosum in three consecutive siblings of a Nigerian family: observations on oculocutaneous manifestations in black African children. Br J Ophthalmol 2001;85:110.

If you would like to post an electronic response to these or any other articles published in the journal, please go to the website, access the article in which you are interested, and click on "eletters: Submit a response to this article" in the box in the top right hand corner. 